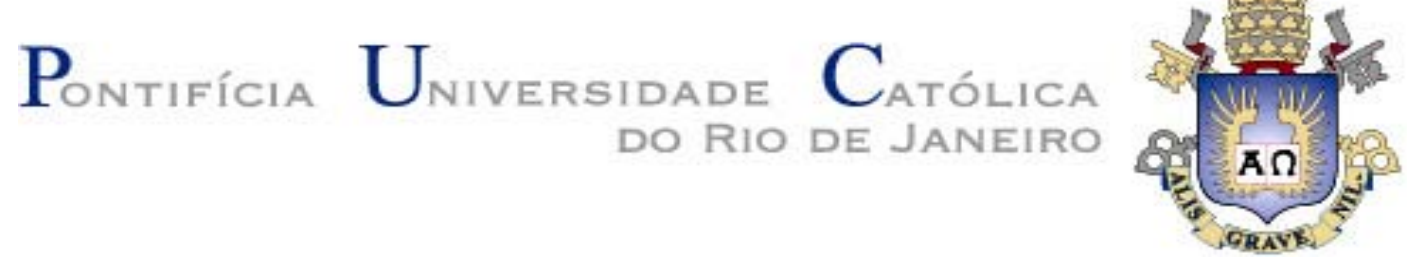

Antonio da Silva Nascimento Filho

Planejamento de Sistemas Móveis em Banda Larga IEEE 802.16e-2005 em Freqüências entre 2 e 11 GHz

Dissertação de Mestrado

Dissertação apresentada ao Programa de Pósgraduação em Engenharia Elétrica da PUC-Rio como requisito parcial para obtenção do título de Mestre em Engenharia Elétrica.

Orientador: Luiz Alencar Reis da Silva Mello

Rio de Janeiro

Setembro de 2008 


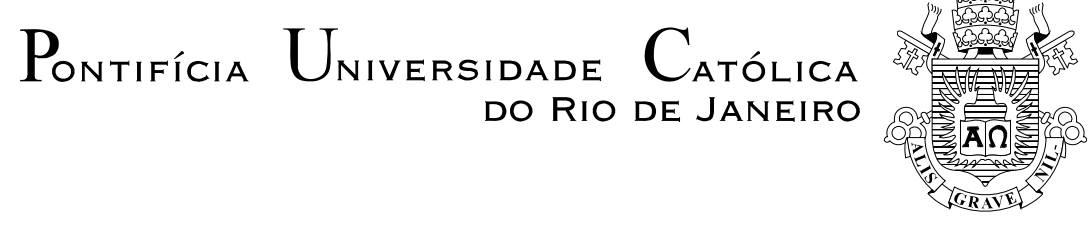

Antonio da Silva Nascimento Filho

\section{Planejamento de Sistemas Móveis em Banda Larga IEEE 802.16e-2005 em Freqüências entre 2 e 11 GHz}

Dissertação apresentada como requisito parcial para obtenção do grau de Mestre pelo Programa de PósGraduação em Engenharia Elétrica da PUC-Rio. Aprovada pela Comissão Examinadora abaixo assinada.

Prof. Luiz Alencar Reis da Silva Mello Orientador Centro de Estudos em Telecomunicações /PUC-Rio

Prof. Rodolfo Sabóia Lima de Souza Centro de Estudos em Telecomunicações /PUC-Rio

Prof. Erasmus Couto Brazil de Miranda UCP

\section{Prof. Rodrigo Martins de Souza Exército Brasileiro}

Prof. José Eugenio Leal Coordenador Setorial do Centro

Técnico Científico - PUC-Rio

Rio de Janeiro, 05 de setembro de 2008 
Todos os direitos reservados. É proibida a reprodução total ou parcial do trabalho sem autorização da universidade, do autor e do orientador.

\section{Antonio da Silva Nascimento Filho}

Graduou-se em Engenharia de Telecomunicações pelo Instituto Militar de Engenharia - IME em 2002. No período de janeiro/2003 a janeiro/2006 foi responsável pela manutenção de sistemas de radiocomunicação em HF do Exército Brasileiro no âmbito do RJ e ES. No período de janeiro/2006 a janeiro/2007 foi responsável pela manutenção de centrais telefônicas do Exército no âmbito do RJ e ES. Desde fevereiro/2007 é administrador de redes de voz e dados do Exército Brasileiro no âmbito do RJ e ES, bem como participa da elaboração de projetos de redes de comunicação via rádio microondas. Suas áreas de interesse são rádio-propagação e sistemas de comunicações móveis.

Ficha Catalográfica

Nascimento Filho, Antonio da Silva

Planejamento de sistemas móveis em banda larga IEEE 802.16e-2005 em freqüências entre 2 e $11 \mathrm{GHz}$ / Antonio da Silva Nascimento Filho; orientador: Luiz Alencar Reis da Silva Mello. - 2008.

199 f. : il. ; $30 \mathrm{~cm}$

Dissertação (Mestrado em Engenharia Elétrica)Pontifícia Universidade Católica do Rio de Janeiro, Rio de Janeiro, 2008.

Inclui bibliografia

1. Engenharia elétrica - Teses. 2. 802.16e-2005. 3. WiMAX móvel. 4. Planejamento de sistemas de comunicações móveis. 5. OFDM. 6. MIMO. 7. Antenas inteligentes. 8. Códigos espaço-temporais. I. Mello, Luiz Alencar Reis da Silva. II. Pontifícia Universidade Católica do Rio de Janeiro. Departamento de Engenharia Elétrica. III. Título. 
Dedico o sucesso desta etapa da minha vida a quem sempre me incentivou a enfrentar desafios, ensinou e esteve presente, nos momentos certos e incertos: a Deus pela graça da vida, aos meus pais e família pelo amor e compreensão e aos amigos por seu apoio e incentivo.

"Tudo que está no plano da realidade já foi sonho um dia." Leonardo da Vinci 


\section{Agradecimentos}

A minha família, pelo incentivo e compreensão durante o período do desenvolvimento deste trabalho e permanente fonte de inspiração.

Ao meu orientador, Professor Luiz A. R. da Silva Mello, pela sua contínua assessoria e apoio pessoal, moral e profissional.

Aos amigos, chefes e subordinados do $2^{\circ}$ CTA (Exército Brasileiro), pelo apoio profissional e amizade.

A todos os amigos e familiares que de uma forma ou de outra me estimularam ou me ajudaram. 


\section{Resumo}

Nascimento Filho, Antonio da Silva; Silva Mello, Luiz Alencar Reis da. Planejamento de Sistemas Móveis em Banda Larga IEEE 802.16e-2005 em Freqüências entre 2 e 11 GHz. Rio de Janeiro, 2008. 199p. Dissertação de Mestrado - Departamento de Engenharia Elétrica, Pontifícia Universidade Católica do Rio de Janeiro.

O projeto de sistemas móveis em banda larga requer um planejamento detalhado devido à necessidade de maximizar a utilização da largura de banda disponível, maximizar a capacidade da rede, garantir requisitos mínimos de disponibilidade e minimizar os custos de implantação e operação da rede. Este trabalho apresenta uma metodologia de projeto a ser empregada em sistemas móveis em banda larga baseados no padrão IEEE 802.16e-2005 (WiMAX móvel). São apresentadas as novas tecnologias empregadas na elaboração do padrão, o modelo de propagação utilizado no cálculo de enlace, o cálculo da interferência co-canal, o cálculo da porcentagem de cobertura de área, o ganho de subcanalização e o cálculo da capacidade do canal. São analisadas abordagens para redução da interferência co-canal, visando maximizar a capacidade da rede e por fim é apresentado um estudo de caso utilizando uma ferramenta computadorizada de planejamento de redes móveis.

\section{Palavras-chave}

802.16e-2005, WiMAX Móvel, Planejamento de Sistemas de Comunicações Móveis, OFDM, MIMO, Antenas Inteligentes, Códigos Espaço-Temporais 


\section{Abstract}

Nascimento Filho, Antonio da Silva; Silva Mello, Luiz Alencar Reis da (Advisor). Mobile Broadband IEEE 802.16e-2005 Systems Planning for Frequencies between 2 and 11 GHz. Rio de Janeiro, 2008. 199p. MSc. Dissertation - Departamento de Engenharia Elétrica, Pontifícia Universidade Católica do Rio de Janeiro

The design of mobile broadband systems requires detailed planning due to necessity of maximizing the use of available bandwidth, network capacity, achieve minimum availability levels and minimize network implementation and operational costs. This work presents a design methodology to be employed in mobile broadband systems based on the IEEE Std 802.16-2005 (Mobile WiMAX). Here are presented the new technologies employed in the standard, the propagation model used for link budget, co-channel interference, coverage area percentage, sub-channelization gain and channel capacity calculations. Approaches for co-channel interference reduction are analyzed aiming to maximize network capacity and at the end a case study is presented using a computerized tool for mobile network planning.

\section{Key words}

802.16e-2005, Mobile WiMAX, Mobile Communication Systems Planning, OFDM, MIMO, Smarte Antennas, Space Time Codes 


\section{Sumário}

ABREVIAÇÕES E SIGLAS

1 INTRODUÇÃO

1.1. REDES DE ACESSO SEM FIO EM BANDA LARGA 24

1.2. OBJETIVOS E ORGANIZAÇÃO DA DISSERTAÇÃO 26

2 DESCRIÇÃO DO PADRÃO IEEE 802.16e-2005 27

2.1. CARACTERÍSTICAS GERAIS 27

2.2. NOVAS TECNOLOGIAS UTILIZADAS 29

2.2.1. OFDM 29

2.2.2. OFDMA 32

2.2.3. OFDMA escalável (S-OFDMA) 36

$\begin{array}{ll}\text { 2.2.4. OFDMA TDD } & 37\end{array}$

2.2.5. Reuso de Freqüência Fracionário 37

2.2.6. Antenas Inteligentes 39

2.2.7. MIMO (MULTIPLE INPUT MULTIPLE OUTPUT) 46

2.2.8. Códigos Espaço-Temporais $\quad 47$

2.3. PRINCIPAIS CARACTERÍSTICAS DO PADRÃO

IEEE 802.16e-2005 51

2.3.1. Suporte MAC à camada física (PHY) 51

2.3.1.1. Duplexação por Divisão do Tempo (TDD) 51

2.3.1.2. Procedimentos para compartilhamento de bandas de freqüência 52

2.3.1.3. Serviço de entrega de dados para rede móvel 53

2.3.2. A Camada Física (PHY) 54

3 PLANEJAMENTO DE SISTEMAS WiMAX MÓVEIS 57

3.1. METODOLOGIA DE PLANEJAMENTO DE COBERTURA 57

3.2. CÁLCULO DE ENLACE 59

3.2.1. Modelo de Propagação de Erceg et al 59 
3.2.2. Requisitos Mínimos dos Receptores 802.16e-2005

64

3.3. CÁLCULO DA INTERFERÊNCIA CO-CANAL 66

3.3.1. Redução da Interferência através da Setorização 74

3.3.1.1. Cálculo da Redução da Interferência Usando

Setorização Tripla $\quad 74$

3.3.1.2. Cálculo da Redução da Interferência Usando

Setorização Sêxtupla 77

3.4. LIMITAÇÃO DO RAIO DEVIDO À DURAÇÃO DO TTG/RTG 80

3.5. CÁLCULO DA PORCENTAGEM DE COBERTURA DE ÁREA

E MARGEM DE DESVANECIMENTO POR SOMBREAMENTO 84

3.6. GANHO DE SUBCANALIZAÇÃO 91

3.7. CÁLCULO DO RAIO DAS CÉLULAS PARA INTERFACE AÉREA WirelessMAN OFDMA PHY 92

3.8. CÁLCULO DA CAPACIDADE DO CANAL 96

4 ESTUDO DE CASO 99

4.1. ESPECIFICAÇÕES TÉCNICAS 99

4.1.1. Especificações técnicas da ERB e da EM utilizadas 100

4.2. CÁLCULO DE ENLACE E RAIO DAS CÉLULAS 101

4.3. PROJETO 102

4.3.1. Configurações do Projeto 102

4.3.1.1. Técnica de Acesso 102

4.3.1.2. Modelo de Propagação 105

4.3.1.3. Configurações da ERB e EM padrões 108

4.3.1.4. Resolução de Cálculo das Células 111

4.3.1.5. Distribuição das Células no Terreno 112

4.3.1.6. Ferramentas de Análise 113

4.3.2. Resultados das Simulações 116

4.3.2.1. Resultados para Rede Tri-Setorizada e

Freqüência Única 116

4.3.2.2. Resultados para Rede Não-Setorizada e

Freqüência Única 121

4.3.2.3. Rede com Setorização Tripla, Razão de Reuso Um e 
Três Canais

4.3.2.4. Rede com Setorização Tripla, Razão de Reuso Três e Nove Canais

5 CONCLUSÕES E SUGESTÕES

6 REFERÊNCIAS BIBLIOGRÁFICAS

ANEXO A ESPECIFICAÇÕES TÉCNICAS DOS RÁDIOS

133

A.1 Estação Base Airspan HiperMAX

133

A.2 Estação Móvel Airspan MiMAX

ANEXO B PROGRAMAS PARA CÁLCULO DA SIR

135

B.1 Cálculo da SIR para um anel interferente, sem setorização

135

B.2 Cálculo da SIR para dois anéis interferentes, sem setorização

137

B.3 Cálculo da SIR para um anel interferente, com setorização tripla e sêxtupla

B.4 Cálculo dos gráficos da SIR para reuso de freqüência unitário

ANEXO C PROGRAMA PARA CÁLCULO DO RAIO DA CÉLULA

ANEXO D PROGRAMA PARA CÁLCULO DO PERCENTUAL

DE COBERTURA DE ÁREA

ANEXO E TABELAS DE CÁLCULO DO RAIO DA CÉLULA

ANEXO F PROGRAMA PARA CÁLCULO DA CAPACIDADE DO CANAL 


\section{Lista de figuras}

Figura 1 - Perfil de Sistema do WiMAX Móvel 28

Figura 2 - Tons OFDM 30

Figura 3 - (a) Resposta do canal e sinal a ser transmitido.

(b)Desvanecimento seletivo em freqüência.

(c) Espectro OFDM é robusto contra desvanecimento seletivo 30

Figura 4 - Exemplo de transmissor OFDM 31

Figura 5 - Arquitetura básica de um sistema OFDM 31

Figura 6 - Subcanais OFDMA 33

Figura 7 - Sinal OFDMA ao longo do tempo e das freqüências 35

Figura 8 - Exemplo de espectro OFDMA 35

Figura 9 - Estrutura das subportadoras (subcanais) OFDMA 36

Figura 10 - Estrutura de um quadro multizona 38

Figura 11 - Reuso de freqüência fracionário $\quad 39$

Figura 12 - Padrão de cobertura setorizada de sistema de feixe comutado $\quad 40$

Figura 13 - Diferença entre uma estratégia comutada e uma adaptativa 41

Figura 14 - Cobertura de um conjunto adaptativo: Lóbulo principal

voltado para usuário e nulo voltado para interferência co-canal 41

Figura 15 - Padrão de cobertura para feixe comutado e

conjunto de antenas adaptativas

Figura 16 - Exemplo de sistema adaptativo atendendo

a dois usuários simultaneamente no mesmo canal

Figura 17 - Comparação entre esquemas de antenas inteligentes

$(\mathrm{a}, \mathrm{b}, \mathrm{c})$ e sistemas MIMO (d)

Figura 18 - Diagrama de um sistema MIMO de transmissão sem fio. 48

Figura 19 - Multiplexação espacial básica com 3 antenas de TX e RX. 49

Figura 20 - Sistema de transmissão com 4 camadas horizontais. $\quad 50$

Figura 21 - Sistema de transmissão com 4 camadas diagonais. 51

Figura 22 - Estrutura do quadro TDD 52

Figura 23 - Estrutura temporal do símbolo OFDMA 55 
Figura 24 - Descrição de freqüências OFDMA (exemplo com 3 canais)

Figura 25 - (a) Plano de Reuso de freqüência $\mathrm{N}=7$;

(b) Sistema celular de 7 grupos

Figura 26 - Interferências em configurações celulares hexagonais

Figura 27 - (a) Distância ao transmissor interferente;

(b) Distância ao transmissor desejado

Figura $28-\mathrm{SIR}$ versus $\mathrm{h}_{\mathrm{B}}$ para $\mathrm{N}=1$ e Terreno Categoria $\mathrm{A}$, sem setorização

Figura 29 - SIR versus $h_{B}$ para $N=1$ e Terreno Categoria $B$, sem setorização

Figura 30 - SIR versus $h_{B}$ para $N=1$ e Terreno Categoria $C$, sem setorização 73

Figura 31 - Interferência com setorização tripla.

Figura $32-\mathrm{SIR}$ versus $\mathrm{h}_{\mathrm{B}}$ para $\mathrm{N}=1$ e Terreno Categoria $\mathrm{A}$, setorização tripla

Figura 33 - SIR versus $h_{B}$ para $N=1$ e Terreno Categoria $B$, setorização tripla

Figura 34 - SIR versus $h_{B}$ para $\mathrm{N}=1$ e Terreno Categoria $C$, setorização tripla 76

Figura 35 - Interferência com setorização sêxtupla.

Figura $36-\mathrm{SIR}$ versus $\mathrm{h}_{\mathrm{B}}$ para $\mathrm{N}=1$ e Terreno Categoria $\mathrm{A}$, setorização sêxtupla

Figura 37 - SIR versus $h_{B}$ para $N=1$ e Terreno Categoria $B$, setorização sêxtupla

Figura 38 - SIR versus $h_{B}$ para $\mathrm{N}=1$ e Terreno Categoria $C$, setorização sêxtupla 79

Figura 39 - Área de cobertura do estudo de caso 99

Figura 40 - Relevo da região em estudo 100

Figura 41 - Configurando os Parâmetros da Técnica de Acesso

Figura 42 - Técnica de Acesso: (a) Largura de banda do sistema e do usuário; (b) Perdas e margens

Figura 43 - Escolha do Modelo de Propagação

Figura 44 - Configuração dos Modelos de Propagação 
Figura 45 - Configuração dos Equipamentos: ERBs e sem 108

Figura 46 - Configurações da ERB padrão 110

Figura 47 - Configurações da EM padrão 111

Figura 48 - Parâmetros gerais dos gráficos das células 111

Figura 49 - Ilustração das ERBs na área de cobertura 112

Figura 50 - Medidor de intensidade de sinal 113

Figura 51 - (a) Gerenciador de Análise; (b) Filtros de Análise;

(c) Configuração dos Gráficos; (d) Configuração dos níveis

de sinal; (e) Resultados da Análise 115

$\begin{array}{ll}\text { Figura } 52 \text { - Análise de melhor servidor } & 117\end{array}$

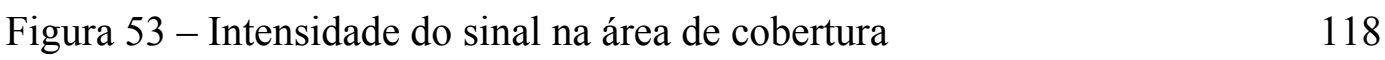

Figura 54 - Cobertura de cada modulação, sem considerar margem de $\begin{array}{ll}\text { desvanecimento } & 118\end{array}$

Figura 55 - Cobertura de cada modulação, considerando a margem de desvanecimento 119

Figura 56 - Análise de interferência entre as células 119

Figura 57 - Análise da relação Sinal-Ruído+Interferência 120

$\begin{array}{ll}\text { Figura } 58 \text { - Análise de melhor servidor } & 121\end{array}$

Figura 59 - Cobertura de cada modulação, considerando a margem de desvanecimento 122

Figura 60 - Análise de interferência entre as células 122

Figura 61 - Análise da relação Sinal-Ruído+Interferência 123

$\begin{array}{ll}\text { Figura } 62 \text { - Alocação de canais em cada célula } & 124\end{array}$

Figura 63 - Análise de interferência entre as células $\quad 124$

Figura 64 - Análise da relação Sinal-Ruído+Interferência 125

Figura 65 - Alocação de canais em cada célula $\quad 126$

Figura 66 - Análise de interferência entre as células 126

Figura 67 - Análise da relação Sinal-Ruído+Interferência 127 


\section{Lista de tabelas}

Tabela 1 - Parâmetros de Escalabilidade do OFDMA 36

Tabela 2 - Características e benefícios de sistemas de antenas inteligentes 45

Tabela 3 - Tipos de serviços de entrega de dados 53

Tabela 4 - Nomenclatura das interfaces aéreas [Tabela 1 do IEEE Std 802.16-2004] 54

Tabela 5 - Parâmetros do Modelo Erceg et al $\quad 61$

Tabela 6 - Relação Sinal Ruído Mínima para interface aérea

WirelessMAN OFDMA

Tabela 7 - Influência do segundo anel interferente $\quad 68$

Tabela 8 - Interferência co-canal no Modelo Erceg et al considerando apenas o $1^{\circ}$ anel interferente para $h_{B}=30 \mathrm{~m} \quad 71$

Tabela 9 - Interferência co-canal no Modelo Erceg et al considerando os $1^{\circ}$ e $2^{\circ}$ anéis interferentes para $h_{B}=30 \mathrm{~m}$

Tabela 10 - Interferência co-canal no Modelo Erceg et al considerando os $1^{\circ}$ e $2^{\circ}$ anéis interferentes para $h_{B}=18 \mathrm{~m}$

Tabela 11 - Interferência co-canal no Modelo Erceg et al com setorização tripla e considerando apenas o $1^{\circ}$ anel interferente para $\mathrm{h}_{\mathrm{B}}=30 \mathrm{~m} \quad 75$

Tabela 12 - Interferência co-canal no Modelo Erceg et al com setorização sêxtupla e considerando apenas o $1^{\circ}$ anel interferente para $\mathrm{h}_{\mathrm{B}}=30 \mathrm{~m} \quad 78$

Tabela 13 - Duração dos intervalos TTG e RTG em termos de um OS 80

Tabela 14 - Cálculo da duração de um slot físico (PS) 81

Tabela 15 - Duração dos intervalos TTG e RTG em $\mu$ s $\quad 81$

Tabela 16 - Valores de $\mathrm{T}_{\text {UL-ERB }}$ para o caso $\mathrm{T}_{\mathrm{DL}-\mathrm{ERB}}=3 \mathrm{~T}_{\mathrm{UL}-\mathrm{ERB}} \quad 84$

Tabela 17 - Raio máximo das células devido à duração do TTG 84

Tabela 18 - Desvio padrão para cálculo do percentual de cobertura de área $\quad 90$

Tabela 19 - Margem para cobertura de área desejada 90 
Tabela 20 - Número de subcanais para alocação PUSC

Tabela 21 - Raio das Células com $\mathrm{f}=2,5 \mathrm{GHz}, \mathrm{CAP}=90 \%$ e alocação PUSC

Tabela 22 - Raio das Células com $\mathrm{f}=3,5 \mathrm{GHz}, \mathrm{CAP}=90 \%$ e alocação PUSC

Tabela 23 - Raio das Células com $\mathrm{f}=2,5 \mathrm{GHz}, \mathrm{CAP}=95 \%$ e alocação PUSC

Tabela 24 - Raio das Células com $\mathrm{f}=3,5 \mathrm{GHz}, \mathrm{CAP}=95 \%$ e alocação PUSC

Tabela 25 - Raio das Células com $\mathrm{f}=2,5 \mathrm{GHz}, \mathrm{CAP}=99 \%$ e alocação PUSC

Tabela 26 - Raio das Células com $\mathrm{f}=3,5 \mathrm{GHz}, \mathrm{CAP}=99 \%$ e alocação PUSC

Tabela 27 - Características da PHY OFDMA conforme o Perfil de Sistema

Tabela 28 - Capacidade máxima do canal para faixa de $2,5 \mathrm{GHz}$, com SNR mínima

Tabela 29 - Capacidade máxima do canal para faixa de 3,5 GHz, com SNR mínima

Tabela 30 - Especificações da Estação Rádio-Base

Tabela 31 - Especificações da Estação Móvel

Tabela 32- Raio das Células com $\mathrm{f}=2,5 \mathrm{GHz}, \mathrm{CAP}=90 \%$ e alocação PUSC

Tabela 33 - Alturas das ERBs do estudo de caso

Tabela 34 - Parâmetros dos modelos de propagação de cada célula

Tabela 35 - Características Técnicas da Estação Base

Airspan HiperMAX

Tabela 36 - Características Técnicas da Estação Móvel

Airspan MiMAX

Tabela 37 - Cálculo de Enlace OFDMA PHY, PUSC,

QPSK $1 / 2, \mathrm{f}=2,5 \mathrm{GHz}, \mathrm{CAP}=90 \%$

Tabela 38 - Cálculo de Enlace OFDMA PHY, PUSC,

QPSK $3 / 4, \mathrm{f}=2,5 \mathrm{GHz}, \mathrm{CAP}=90 \%$ 
Tabela 39 - Cálculo de Enlace OFDMA PHY, PUSC,

16-QAM 1/2, $\mathrm{f}=2,5 \mathrm{GHz}, \mathrm{CAP}=90 \%$

Tabela 40 - Cálculo de Enlace OFDMA PHY, PUSC, 16-QAM 3/4, f=2,5 GHz, CAP $=90 \%$

Tabela 41 - Cálculo de Enlace OFDMA PHY, PUSC,

$$
\text { 64-QAM 1/2, } \mathrm{f}=2,5 \mathrm{GHz}, \mathrm{CAP}=90 \%
$$

Tabela 42 - Cálculo de Enlace OFDMA PHY, PUSC,

$$
\text { 64-QAM 2/3, } \mathrm{f}=2,5 \mathrm{GHz}, \mathrm{CAP}=90 \%
$$

Tabela 43 - Cálculo de Enlace OFDMA PHY, PUSC,

$$
\text { 64-QAM 3/4, } \mathrm{f}=2,5 \mathrm{GHz}, \mathrm{CAP}=90 \%
$$

Tabela 44 - Cálculo de Enlace OFDMA PHY, PUSC,

$$
\text { 64-QAM 5/6, f = 2,5 GHz, CAP }=90 \%
$$

Tabela 45 - Cálculo de Enlace OFDMA PHY, PUSC,

$$
\text { QPSK } 1 / 2, \mathrm{f}=3,5 \mathrm{GHz}, \mathrm{CAP}=90 \%
$$

Tabela 46 - Cálculo de Enlace OFDMA PHY, PUSC,

$$
\text { QPSK 3/4, } \mathrm{f}=3,5 \mathrm{GHz}, \mathrm{CAP}=90 \%
$$

Tabela 47 - Cálculo de Enlace OFDMA PHY, PUSC,

$$
\text { 16-QAM 1/2, } \mathrm{f}=3,5 \mathrm{GHz}, \mathrm{CAP}=90 \%
$$

Tabela 48 - Cálculo de Enlace OFDMA PHY, PUSC,

$$
\text { 16-QAM 3/4, } \mathrm{f}=3,5 \mathrm{GHz}, \mathrm{CAP}=90 \%
$$

Tabela 49 - Cálculo de Enlace OFDMA PHY, PUSC,

$$
\text { 64-QAM 1/2, } \mathrm{f}=3,5 \mathrm{GHz}, \mathrm{CAP}=90 \%
$$

Tabela 50 - Cálculo de Enlace OFDMA PHY, PUSC,

$$
\text { 64-QAM 2/3, f = 3,5 GHz, CAP }=90 \%
$$

Tabela 51 - Cálculo de Enlace OFDMA PHY, PUSC,

$$
\text { 64-QAM 3/4, f =3,5 GHz, CAP }=90 \%
$$

Tabela 52 - Cálculo de Enlace OFDMA PHY, PUSC,

$$
\text { 64-QAM 5/6, f = 3,5 GHz, CAP }=90 \%
$$

Tabela 53 - Cálculo de Enlace OFDMA PHY, PUSC,

QPSK $1 / 2, \mathrm{f}=2,5 \mathrm{GHz}, \mathrm{CAP}=95 \%$

Tabela 54 - Cálculo de Enlace OFDMA PHY, PUSC,

QPSK $3 / 4, \mathrm{f}=2,5 \mathrm{GHz}, \mathrm{CAP}=95 \%$

Tabela 55 - Cálculo de Enlace OFDMA PHY, PUSC, 


$$
\text { 16-QAM 1/2, } \mathrm{f}=2,5 \mathrm{GHz}, \mathrm{CAP}=95 \%
$$

Tabela 56 - Cálculo de Enlace OFDMA PHY, PUSC,

$$
\text { 16-QAM 3/4, } \mathrm{f}=2,5 \mathrm{GHz}, \mathrm{CAP}=95 \%
$$

Tabela 57 - Cálculo de Enlace OFDMA PHY, PUSC,

$$
\text { 64-QAM 1/2, } \mathrm{f}=2,5 \mathrm{GHz}, \mathrm{CAP}=95 \%
$$

Tabela 58 - Cálculo de Enlace OFDMA PHY, PUSC,

$$
\text { 64-QAM 2/3, } \mathrm{f}=2,5 \mathrm{GHz}, \mathrm{CAP}=95 \%
$$

Tabela 59 - Cálculo de Enlace OFDMA PHY, PUSC,

$$
\text { 64-QAM 3/4, f = 2,5 GHz, CAP }=95 \%
$$

Tabela 60 - Cálculo de Enlace OFDMA PHY, PUSC,

$$
\text { 64-QAM 5/6, f= 2,5 GHz, CAP = } 95 \%
$$

Tabela 61 - Cálculo de Enlace OFDMA PHY, PUSC,

$$
\text { QPSK } 1 / 2, \mathrm{f}=3,5 \mathrm{GHz}, \mathrm{CAP}=95 \%
$$

Tabela 62 - Cálculo de Enlace OFDMA PHY, PUSC,

$$
\text { QPSK } 3 / 4, \mathrm{f}=3,5 \mathrm{GHz}, \mathrm{CAP}=95 \%
$$

Tabela 63 - Cálculo de Enlace OFDMA PHY, PUSC,

$$
\text { 16-QAM } 1 / 2, \mathrm{f}=3,5 \mathrm{GHz}, \mathrm{CAP}=95 \%
$$

Tabela 64 - Cálculo de Enlace OFDMA PHY, PUSC,

$$
\text { 16-QAM 3/4, } \mathrm{f}=3,5 \mathrm{GHz}, \mathrm{CAP}=95 \%
$$

Tabela 65 - Cálculo de Enlace OFDMA PHY, PUSC,

$$
\text { 64-QAM 1/2, } \mathrm{f}=3,5 \mathrm{GHz}, \mathrm{CAP}=95 \%
$$

Tabela 66 - Cálculo de Enlace OFDMA PHY, PUSC,

$$
\text { 64-QAM 2/3, } \mathrm{f}=3,5 \mathrm{GHz}, \mathrm{CAP}=95 \%
$$

Tabela 67 - Cálculo de Enlace OFDMA PHY, PUSC,

$$
\text { 64-QAM 3/4, } \mathrm{f}=3,5 \mathrm{GHz}, \mathrm{CAP}=95 \%
$$

Tabela 68 - Cálculo de Enlace OFDMA PHY, PUSC,

$$
\text { 64-QAM 5/6, } \mathrm{f}=3,5 \mathrm{GHz}, \mathrm{CAP}=95 \%
$$

Tabela 69 - Cálculo de Enlace OFDMA PHY, PUSC,

QPSK $1 / 2, \mathrm{f}=2,5 \mathrm{GHz}, \mathrm{CAP}=99 \%$

Tabela 70 - Cálculo de Enlace OFDMA PHY, PUSC,

QPSK $3 / 4, \mathrm{f}=2,5 \mathrm{GHz}, \mathrm{CAP}=99 \%$

Tabela 71 - Cálculo de Enlace OFDMA PHY, PUSC,

$$
16-\mathrm{QAM} 1 / 2, \mathrm{f}=2,5 \mathrm{GHz}, \mathrm{CAP}=99 \%
$$


Tabela 72 - Cálculo de Enlace OFDMA PHY, PUSC,

16-QAM 3/4, $\mathrm{f}=2,5 \mathrm{GHz}, \mathrm{CAP}=99 \%$

Tabela 73 - Cálculo de Enlace OFDMA PHY, PUSC, 64-QAM 1/2, $\mathrm{f}=2,5 \mathrm{GHz}, \mathrm{CAP}=99 \%$

Tabela 74 - Cálculo de Enlace OFDMA PHY, PUSC,

$$
\text { 64-QAM 2/3, f = 2,5 GHz, CAP = } 99 \%
$$

Tabela 75 - Cálculo de Enlace OFDMA PHY, PUSC,

$$
\text { 64-QAM 3/4, } \mathrm{f}=2,5 \mathrm{GHz}, \mathrm{CAP}=99 \%
$$

Tabela 76 - Cálculo de Enlace OFDMA PHY, PUSC,

$$
\text { 64-QAM 5/6, f = 2,5 GHz, CAP }=99 \%
$$

Tabela 77 - Cálculo de Enlace OFDMA PHY, PUSC,

QPSK $1 / 2, \mathrm{f}=3,5 \mathrm{GHz}, \mathrm{CAP}=99 \%$

Tabela 78 - Cálculo de Enlace OFDMA PHY, PUSC,

$$
\text { QPSK 3/4, } \mathrm{f}=3,5 \mathrm{GHz}, \mathrm{CAP}=99 \%
$$

Tabela 79 - Cálculo de Enlace OFDMA PHY, PUSC, 16-QAM 1/2, $\mathrm{f}=3,5 \mathrm{GHz}, \mathrm{CAP}=99 \%$

Tabela 80 - Cálculo de Enlace OFDMA PHY, PUSC, 16-QAM 3/4, f=3,5 GHz, CAP = $99 \%$

Tabela 81 - Cálculo de Enlace OFDMA PHY, PUSC,

$$
\text { 64-QAM 1/2, } \mathrm{f}=3,5 \mathrm{GHz}, \mathrm{CAP}=99 \%
$$

Tabela 82 - Cálculo de Enlace OFDMA PHY, PUSC,

$$
\text { 64-QAM 2/3, } \mathrm{f}=3,5 \mathrm{GHz}, \mathrm{CAP}=99 \%
$$

Tabela 83 - Cálculo de Enlace OFDMA PHY, PUSC,

$$
\text { 64-QAM 3/4, } \mathrm{f}=3,5 \mathrm{GHz}, \mathrm{CAP}=99 \%
$$

Tabela 84 - Cálculo de Enlace OFDMA PHY, PUSC,

$$
\text { 64-QAM 5/6, f=3,5 GHz, CAP = } 99 \%
$$




\section{ABREVIAÇÕES E SIGLAS}

\begin{tabular}{|c|c|}
\hline 3-DES & triple data encryption standard \\
\hline AAS & adaptive antenna system \\
\hline $\mathrm{AGC}$ & automatic gain control \\
\hline $\mathrm{AK}$ & authorization key \\
\hline ARQ & automatic repeat request \\
\hline ATDD & adaptive time division duplexing \\
\hline ATM & asynchronous transfer mode \\
\hline $\mathrm{BCC}$ & block convolutional code \\
\hline $\mathrm{BE}$ & best effort \\
\hline BER & bit error rate \\
\hline BPSK & binary phase shift keying \\
\hline $\mathrm{BR}$ & bandwidth request \\
\hline $\mathrm{BS}$ & base station \\
\hline $\mathrm{BSN}$ & block sequence number \\
\hline BTC & block turbo code \\
\hline $\mathrm{BW}$ & bandwidth \\
\hline BWA & broadband wireless access \\
\hline BWSU & broadband wireless subscriber unit \\
\hline $\mathrm{C} / \mathrm{I}$ & carrier-to-interference ratio \\
\hline $\mathrm{C} / \mathrm{N}$ & carrier-to-noise ratio \\
\hline CA & certification authority \\
\hline $\mathrm{CBC}$ & cipher block chaining \\
\hline $\mathrm{CC}$ & confirmation code \\
\hline $\mathrm{CCI}$ & co-channel interference \\
\hline CCS & common channel signaling \\
\hline $\mathrm{CCV}$ & clock comparison value \\
\hline CDMA & code division multiple access \\
\hline CEPT & $\begin{array}{l}\text { european conference of postal and telecommunications } \\
\text { administrations }\end{array}$ \\
\hline ChID & channel identifier \\
\hline CID & connection identifier \\
\hline CINR & carrier-to-interference-and-noise ratio \\
\hline CIR & channel impulse response \\
\hline CLP & cell loss priority \\
\hline $\mathrm{CP}$ & cyclic prefix \\
\hline CPS & common part sublayer \\
\hline CRC & cyclic redundancy check \\
\hline $\mathrm{CS}$ & convergence sublayer \\
\hline $\mathrm{CSCF}$ & centralized scheduling configuration \\
\hline $\mathrm{CSCH}$ & centralized scheduling \\
\hline DAMA & demand assigned multiple access \\
\hline DARS & digital audio radio satellite \\
\hline
\end{tabular}




\begin{tabular}{|c|c|}
\hline DCD & downlink channel descripto \\
\hline DES & data encryption standard \\
\hline DFS & dynamic frequency selection \\
\hline DHCP & dynamic host configuration protocol \\
\hline DIUC & downlink interval usage code \\
\hline DL & downlink \\
\hline DM & directed mesh \\
\hline DSA & dynamic service addition \\
\hline DSC & dynamic service change \\
\hline $\mathrm{DSCH}$ & distributed scheduling \\
\hline DSCP & differentiated services codepoint \\
\hline DSD & dynamic service deletion \\
\hline DSx & dynamic service addition, change, or deletion \\
\hline $\mathrm{EC}$ & encryption control \\
\hline $\mathrm{ECB}$ & electronic code book \\
\hline EDE & encrypt-decrypt-encrypt \\
\hline EESS & earth exploratory satellite system \\
\hline EIRP & effective isotropic radiated power \\
\hline EKS & encryption key sequence \\
\hline EVM & error vector magnitude \\
\hline $\mathrm{FC}$ & fragmentation control \\
\hline $\mathrm{FCH}$ & frame control header \\
\hline FDD & frequency division duplex or duplexing \\
\hline FEC & forward error correction \\
\hline FFT & fast fourier transform \\
\hline FPC & fast power control \\
\hline FSH & fragmentation subheader \\
\hline FSN & fragment sequence number \\
\hline FSS & fixed satellite service \\
\hline FWA & fixed wireless access \\
\hline GF & galois field \\
\hline GPS & global positioning system \\
\hline GS & guard symbol \\
\hline $\mathrm{HCS}$ & header check sequence \\
\hline HEC & header error check \\
\hline H-FDD & half-duplex frequency division duplex \\
\hline HMAC & hashed message authentication code \\
\hline HT & header type \\
\hline HUMAN & high-speed unlicensed metropolitan area network \\
\hline $\mathrm{I}$ & inphase \\
\hline IANA & internet assigned numbers authority \\
\hline IE & information element \\
\hline IFFT & inverse fast fourier transform \\
\hline IP & internet protocol \\
\hline ITU & international telecommunications union \\
\hline IWF & interworking function \\
\hline KEK & key encryption key \\
\hline LAN & local area network \\
\hline LFSR & linear feedback shift register \\
\hline LLC & logical link control \\
\hline
\end{tabular}




\begin{tabular}{|c|c|}
\hline LOS & line-of-sight \\
\hline LSB & least significant bit \\
\hline MAC & medium access control layer \\
\hline MAN & metropolitan area network \\
\hline $\mathrm{MBd}$ & megabaud \\
\hline $\mathrm{MBd} / \mathrm{s}$ & megabaud per second \\
\hline $\mathrm{Mb} / \mathrm{s}$ & megabit per second \\
\hline MDS & multipoint distribution service \\
\hline MIB & management information base \\
\hline MIC & message integrity check \\
\hline MMDS & multichannel multipoint distribution service \\
\hline MPEG & moving pictures experts group \\
\hline MSB & most significant bit \\
\hline MSH & mesh \\
\hline NCFG & network configuration \\
\hline NENT & network entry \\
\hline NLOS & non-line-of-sight \\
\hline NNI & network-to-network interface (or network node interface) \\
\hline nrtPS & non-real-time polling service \\
\hline OFDM & orthogonal frequency division multiplexing \\
\hline OFDMA & orthogonal frequency division multiple access \\
\hline OID & object identifier \\
\hline PBR & piggyback request \\
\hline PDU & protocol data unit \\
\hline PHS & payload header suppression \\
\hline PHSF & payload header suppression field \\
\hline PHSI & payload header suppression index \\
\hline PHSM & payload header suppression mask \\
\hline PHSS & payload header suppression size \\
\hline PHSV & payload header suppression valid \\
\hline PHY & physical layer \\
\hline PKM & privacy key management \\
\hline PM & poll-me bit \\
\hline PMD & physical medium dependent \\
\hline PMP & point-to-multipoint \\
\hline PPP & point-to-point protocol \\
\hline PRBS & pseudo-random binary sequence \\
\hline PS & physical slot \\
\hline PSH & packing subheader \\
\hline PTI & payload type indicator \\
\hline $\mathrm{PtP}$ & point to point \\
\hline PVC & permanent virtual circuit \\
\hline Q & quadrature \\
\hline QAM & quadrature amplitude modulation \\
\hline QoS & quality of service \\
\hline QPSK & quadrature phase-shift keying \\
\hline REQ & request \\
\hline RLAN & radio local access network \\
\hline RNG & ranging \\
\hline $\mathrm{RS}$ & Reed-Solomon \\
\hline
\end{tabular}


RSL

RSP

RSS

RSSI

RTG

rtPS

$\mathrm{Rx}$

RxDS

SA

SAID

SAP

SAR

$\mathrm{SC}$

SCTE

SDU

SF

SFID

SHA

SI

SLA

SLPID - Sleep ID

received signal level

response

receive signal strength

receive signal strength indicator

receive/transmit transition gap. This is a gap between the last sample of the uplink burst and the first sample of the subsequent downlink burst at the antenna port of the base station (BS) in a time division duplex (TDD) transceiver. This gap allows time for the base station (BS) to switch from receive to transmit mode. During this gap, the BS is not transmitting modulated data but simply allowing the BS transmitter carrier to ramp up, and the transmit/receive $(\mathrm{Tx} / \mathrm{Rx})$ antenna switch to actuate. Not applicable for frequency division duplex (FDD) systems.

real-time polling service

receiver

receiver delay spread clearing interval

security association

security association identifier

service access point

synthetic aperture radar

single carrier

society of cable telecommunications engineers

service data unit

service flow

service flow identifier

secure hash algorithm

slip indicator

service level agreement

This is a number assigned by the BS whenever an MS is instructed to enter sleep mode. This number shall be unique in the sense that it is assigned to a single MS that is instructed to enter sleep mode. No other MS shall be assigned the same number while the first MS is still in sleep mode.

SNMP

simple network management protocol

SNR

SS

SSTG

SSTTG

SSRTG

STC

SVC

TC

TCM

TCP

TDD

TDM

TDMA

TEK

subscriber station

subscriber station transition gap

subscriber station tx/rx gap

subscriber station rx/tx gap

space time coding

switched virtual circuit

transmission convergence sublayer

trellis coded modulation

transmission control protocol

time division duplex or duplexing

time division multiplexing

time division multiple access

traffic encryption key 
TFTP

TLV

TTG

Tx

UCD

UDP

UGS

UIUC

UL

UNI

U-NII

UTC

UW

$\mathrm{VC}$

VCI

VLAN

VP

VPI

WirelessMAN

WirelessHUMAN

WPOW

XOR trivial file transfer protocol

type/length/value

transmit/receive transition gap. This is a gap between the last sample of the downlink burst and the first sample of the subsequent uplink burst at the antenna port of the base station (BS) in a time division duplex (TDD) transceiver. This gap allows time for the base station (BS) to switch from transmit to receive mode. During this gap, the BS is not transmitting modulated data but simply allowing the BS transmitter carrier to ramp down, the transmit/receive (Tx/Rx) antenna switch to actuate, and the BS receiver section to activate. Not applicable for frequency division duplex (FDD) systems.

transmitter

uplink channel descriptor

user datagram protocol

unsolicited grant service

uplink interval usage code

uplink

user-to-network interface or user-network interface

unlicensed national information infrastructure

universal coordinated time

unique word

virtual channel

virtual channel identifier

virtual local area network

virtual path

virtual path identifier

Wireless Metropolitan Area Networks

Wireless High-speed Unlicensed Metropolitan Area

Networks

Wings Planner On the Web

exclusive-or 